\title{
CP violation with the ATLAS detector
}

\author{
David Rousseau ${ }^{\mathrm{a}}$, contribution to the III ${ }^{\mathrm{rd}}$ Conference on Hyperons, Charms and Beauty Hadrons, \\ Genova, 1998, to be published in Nuclear Physics B
}

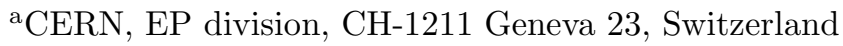

Measurements of CP violation parameters in the B system with the ATLAS detector at LHC are reviewed. Measurements of $\sin 2 \beta$ in the $\mathrm{B}_{\mathrm{d}}^{0} \rightarrow \mathrm{J} / \psi \mathrm{K}_{\mathrm{S}}^{0}$ channel and of $\sin 2 \alpha$ in the $\mathrm{B}_{\mathrm{d}}^{0} \rightarrow \pi^{+} \pi^{-}$channel are described in details. Other channels sensitive to the angles $\gamma$ and $\delta \gamma$ are also presented.

\section{Introduction}

At the LHC approximately $10^{12} \mathrm{~b} \overline{\mathrm{b}}$ pair will be produced per year and per experiment during the first 3 years of running at low luminosity $\left(10^{33} \mathrm{~cm}^{-2} \mathrm{~s}^{-1}\right)$. This will provide the opportunity for the ATLAS experiment to perform accurate measurement of some of the Unitarity Triangle parameters. The design of the ATLAS detector is essentially frozen. The full GEANT description of the detector has been tuned to reproduce test-beam data, and passive material in the simulation updated to the engineering design. Several B-physics simulation, first presented in the ATLAS technical proposal [3], are being updated and improved $[4,5]$, with this more realistic simulation and new experimental inputs. An overview of the most recent simulation results is presented here. This talk concentrates on $\mathrm{CP}$ violation. The ATLAS detector, particle identification and reconstruction, and the measurement of other parameters are described in Ref. [2].

Results are given for one year of running at low luminosity, corresponding to an integrated luminosity of $10^{4} \mathrm{pb}^{-1}$.

\section{2. $\sin 2 \beta$ : the $\mathrm{J} / \psi \mathrm{K}_{\mathrm{S}}^{0}$ channel}

The decay $\mathrm{B}_{\mathrm{d}}^{0} \rightarrow \mathrm{J} / \psi \mathrm{K}_{\mathrm{S}}^{0}$ is generally considered to be the gold-plated channel for measuring the angle $\beta$. It is theoretically clean, with penguin diagrams contributing by less than $1 \%$. The timedependent CP asymmetry has the following form:

$$
A(t)=\sin 2 \beta \sin \left(x_{\mathrm{d}} t / \tau_{\mathrm{B}_{\mathrm{d}}^{0}}\right) .
$$

The current standard model best fit of the Unitarity Triangle yields $\sin 2 \beta=0.68 \pm 0.1[6]$.

\subsection{Trigger}

The B physics trigger in ATLAS relies on a $6 \mathrm{GeV} / \mathrm{c} p_{\mathrm{T}}$ muon trigger at Level 1 [2]. Full reconstruction of the event and lepton identification is done at Level 2: muons are identified with $p_{\mathrm{T}}$ down to $5 \mathrm{GeV} / \mathrm{c}$ using the muon spectrometer (identification down to $3 \mathrm{GeV} / \mathrm{c}$ using the hadron calorimeter is being studied), electrons are identified down to $p_{\mathrm{T}}=1 \mathrm{GeV} / \mathrm{c}$ using the transition radiation in the TRT.

Two $\mathrm{J} / \psi$ samples are available: the $\mathrm{J} / \psi \rightarrow \mathrm{e}^{+} \mathrm{e}^{-}$ sample, with a trigger on the muon from the accompanying $\mathrm{B}$ hadron, and the self-triggering $\mathrm{J} / \psi \rightarrow \mu^{+} \mu^{-}$sample. This sample is more copious, but initial state tagging is more difficult.

\subsection{Event reconstruction}

The $\mathrm{J} / \psi$ is reconstructed from two opposite sign identified leptons, forming a good $3 \mathrm{D}$ vertex separated from the beam-line. The $\mathrm{J} / \psi$ and the identified $\mathrm{K}_{\mathrm{S}}^{0}[2]$ are then combined to form a $\mathrm{B}$ candidate, with good vertex probability (see Ref. [5] for details). The $\mathrm{B}_{\mathrm{d}}^{0}$ mass plot is shown in Fig. 1. The mass resolution is $22 \mathrm{MeV} / \mathrm{c}^{2}$ and a mass cut of three standard deviations is applied.

\subsection{Background}

The dominant combinatorial background is the combination of a true $\mathrm{J} / \psi$ from $\mathrm{B}$ decay, with a $\mathrm{K}_{\mathrm{S}}^{0}$ from fragmentation. The contribution of fake $\mathrm{J} / \psi$ (lepton-lepton, lepton-hadron, hadronhadron) and fake $K_{S}^{0}$ is small. The contribution of direct $\mathrm{J} / \psi$ is removed by the displaced vertex requirement. Backgrounds cause a dilution of the asymmetry: $D_{b k g}=N_{S} /\left(N_{S}+N_{B}\right)$, where $N_{S}$ $\left(N_{B}\right)$ is the number of signal (background) events. 


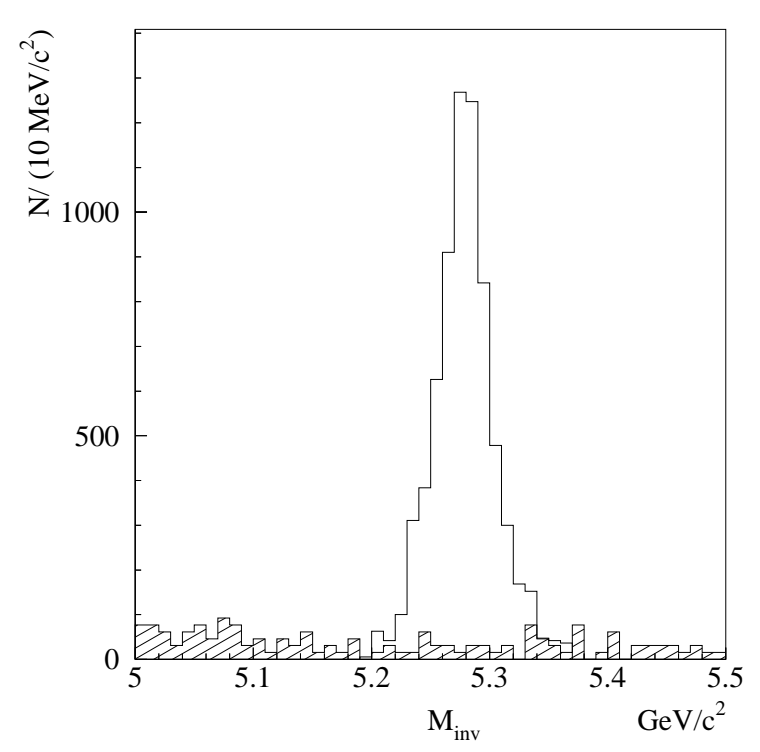

Figure 1. $\mathrm{J} / \psi \mathrm{K}_{\mathrm{S}}^{0}$ mass spectrum, with signal (white) and backgrounds (hatched).

\subsection{Initial state tagging and dilutions}

The tagging of the initial state is necessary to distinguish $\mathrm{B}_{\mathrm{d}}^{0}$ from $\overline{\mathrm{B}}_{\mathrm{d}}^{0}$. Any tagging algorithm is characterized by its efficiency $\epsilon=\left(N^{+}+N^{-}\right) / N_{S}$ and its mistag fraction $W=N^{-} /\left(N^{+}+N^{-}\right)$, where $N^{+}\left(N^{-}\right)$is the number of correctly (incorrectly) tagged signal events. The tagging dilution is $D_{t a g}=1-2 W$. Finally, the tagging quality factor is $Q=\epsilon D_{t a g}^{2}$, which is the equivalent loss in luminosity compared to a perfect tag with $D=\epsilon=1$.

The time-integration of the $\mathrm{CP}$ asymmetry is an additional source of dilution: $D_{\text {int }}=$ $\left(\sin \left(x t_{0} / \tau\right)+x \cos \left(x t_{0} / \tau\right)\right) /\left(1+x^{2}\right)$, where $t_{0}$ is the minimum accepted proper-time and $x$ the usual mixing parameter. If a fit to the timedependent asymmetry is performed, $D_{\text {int }}$ is replaced by $D_{\text {fit }}$ which is one for a perfect time resolution and no threshold on the proper-time.

Finally, the observed asymmetry is:

$A_{o b s}=D_{t a g} D_{b k g} D_{i n t} \sin 2 \beta$, and the sensitivity,

$\delta(\sin 2 \beta)=\frac{\sqrt{1-A_{o b s}^{2}}}{D_{\text {tag }} D_{\text {int }} \sqrt{D_{b k g} N_{S}}}$.

\subsection{Tagging algorithms}

The lepton tagging uses the correlation between the flavour of the $\mathrm{B}_{\mathrm{d}}^{0}$ and the charge of the lepton from the decay of the second $B$ hadron in the event. The mistag fraction of $22 \%$ is partly due to the mixing of the second B hadron, partly to the cascade $\mathrm{b} \rightarrow \mathrm{c} \rightarrow \ell$ decays. The efficiency is $100 \%$ for the $\mathrm{J} / \psi \rightarrow \mathrm{e}^{+} \mathrm{e}^{-}$sample where the trigger muon is used for tagging, but only $5 \%$ in the $\mathrm{J} / \psi \rightarrow \mu^{+} \mu^{-}$sample, where an additional lepton with $p_{\mathrm{T}}>5 \mathrm{GeV} / \mathrm{c}$ is searched for.

Two additional algorithms can be used, especially in the $\mathrm{J} / \psi \rightarrow \mu^{+} \mu^{-}$channel, to supplement the absence of tagging lepton. The B- $\pi$ algorithm uses the correlation between the flavour of the B meson and the charge of the adjacent hadron in the fragmentation chain. The same side-jet algorithm uses the charge correlation between the primary b quark and the nearby fragmentation tracks. Both algorithms have similar performance and are highly correlated, so that, up to now, only one can be used at a time. Their high mistag fraction compared to the lepton tag is compensated by a much higher efficiency $[5,7]$.

Table 1

$\mathrm{J} / \psi \mathrm{K}_{\mathrm{S}}^{0}$ yields and $\sin 2 \beta$ sensitivity.

\begin{tabular}{ccc}
\hline Channel & $\mathrm{e}^{+} \mathrm{e}^{-}$ & $\mu^{+} \mu^{-}$ \\
\hline $\mathrm{N}\left(\mathrm{B}_{\mathrm{d}}^{0} \rightarrow \mathrm{J} / \psi \mathrm{K}_{\mathrm{S}}^{0}\right)$ & 7000 & 213000 \\
$\mathrm{~N}$ background & 480 & 3600 \\
\hline$\delta(\sin 2 \beta)$ lepton tag & 0.036 & 0.029 \\
$\delta(\sin 2 \beta)$ B- $\pi$ tag & 0.16 & 0.025 \\
\hline$\delta(\sin 2 \beta)$ time fit & 0.026 & 0.017 \\
\hline
\end{tabular}

\subsection{Results}

The yields and sensitivity are shown in Table 1 , for an input value $\sin 2 \beta=0.6$. The last line shows the sensitivity of a time-dependent fit combining the two tags. The two samples combined 
yield a final sensitivity of

$\delta_{\text {stat }}(\sin 2 \beta)=0.014$.

The sensitivity degrades to 0.017 if muon identification in the $p_{\mathrm{T}}$ range $3-5 \mathrm{GeV} / \mathrm{c}$ cannot be performed at trigger Level 2. The systematic uncertainties are expected to be of order 0.01[3]. The production and detection asymmetry and the tagging performances will be measured on control channels such as $\mathrm{B}_{\mathrm{d}}^{0} \rightarrow \mathrm{J} / \psi \mathrm{K}^{* 0}$ and $\mathrm{B}^{+} \rightarrow$ $\mathrm{J} / \psi \mathrm{K}^{+}$where no asymmetry is expected. Lowering the electron minimum $p_{\mathrm{T}}$ to $0.5 \mathrm{GeV} / \mathrm{c}$ and improvement to tagging are under study.

\section{3. $\sin 2 \alpha$ : the $\pi^{+} \pi^{-}$channel}

The decay asymmetry in the $\mathrm{B}_{\mathrm{d}}^{0} \rightarrow \pi^{+} \pi^{-}$channel is related to the angle $\alpha$ :

$A(t)=a \cos \left(x_{\mathrm{d}} t / \tau_{\mathrm{B}_{\mathrm{d}}^{0}}\right)+b \sin \left(x_{\mathrm{d}} t / \tau_{\mathrm{B}_{\mathrm{d}}^{0}}\right)$

with, $a$, the direct $\mathrm{CP}$ violation component,

$a=-2 \frac{A_{P}}{A_{T}} \sin \delta \sin \alpha$

and, $b$, the mixing-induced component,

$b=-\sin 2 \alpha-2 \frac{A_{P}}{A_{T}} \cos \delta \cos 2 \alpha \sin \alpha$,

where $A_{P} / A_{T}$ is the ratio of the penguin and tree amplitude, $\delta$ is the penguin and tree amplitude phase difference. It is assumed that $\alpha=$ $\pi-(\beta+\gamma)$. Since there are three unknowns and only two observables ( $a$ and $b$ ), and since $\delta$ is not believed to be calculable, a theoretical estimation of $A_{P} / A_{T}$ is needed. Much theoretical work is currently going on this subject, to which measurements from the near-future B-factory will be valuable inputs. To set the scale, Fleischer and Mannel [8] estimate that, with the help of the measurement of the $\mathrm{B}^{+} \rightarrow \pi^{+} \mathrm{K}^{0}$ and $\mathrm{B}^{+} \rightarrow \pi^{+} \pi^{0}$ branching ratios, the theoretical uncertainty on the penguin contribution would yield an uncertainty on $\alpha$ of less than $3^{\circ}$. The current standard model best fit of the Unitarity Triangle yields the estimate $\sin 2 \alpha=-0.1 \pm 0.40$ [6]. A small value of $\sin 2 \alpha$ could mean no observable CP asymmetry in this channel, but tight bounds on $\sin 2 \alpha$ would in any case be valuable constraint to the Unitarity Triangle.

\subsection{Trigger}

After the Level 1 muon trigger, $\mathrm{B}_{\mathrm{d}}^{0} \rightarrow \pi^{+} \pi^{-}$ candidates are searched for at Level 2 by combining opposite sign particles with $p_{\mathrm{T}}>5.5 \mathrm{GeV} / \mathrm{c}$ and requiring the invariant mass of the pair to be in the range $4.5-6.5 \mathrm{GeV} / \mathrm{c}^{2}$. Lowering as much as possible the minimum pion $p_{\mathrm{T}}$ to significantly increase the event yield is being studied. A value of $4 \mathrm{GeV} / \mathrm{c}$ has been retained in the following, allowing a doubling of the statistics.

\subsection{Reconstruction}

The offline selection requires two charged particles with $p_{\mathrm{T}}>4 \mathrm{GeV} / \mathrm{c}$, separated from the beam-line by more than three standard deviations and forming a good vertex. The $\mathrm{B}_{\mathrm{d}}^{0}$ vertex, beam-line and flight direction should be consistent. To remove the main background where the tracks come from two different $\mathrm{B}$ hadrons, each track is required to be separated from the triggering muon. Finally, a mass cut of one standard deviation $\left( \pm 70 \mathrm{MeV} / \mathrm{c}^{2}\right)$ is applied.

\subsection{Backgrounds}

The list of background branching ratios and yields is shown in Table 2. The combinatorial background can be kept small. Since it is caused by random coincidence of high impact parameter tracks, the use of full simulation and reconstruction is mandatory to measure accurately the tracking resolution, including tails. A high statistic fast simulation is then used to demonstrate

Table 2

Branching ratios and yields in the $\mathrm{B}_{\mathrm{d}}^{0} \rightarrow \pi^{+} \pi^{-}$ channel.

\begin{tabular}{ccc}
\hline Channel & BR's & Yield \\
\hline $\mathrm{B}_{\mathrm{d}}^{0} \rightarrow \pi^{+} \pi^{-}$ & $0.7 \times 10^{-5}$ & 2500 \\
\hline $\mathrm{B}_{\mathrm{d}}^{0} \rightarrow \mathrm{K}^{+} \pi^{-}$ & $1.5 \times 10^{-5}$ & 4600 \\
$\mathrm{~B} \rightarrow \rho \pi^{-}$ & $0.7 \times 10^{-5}$ & 70 \\
$\mathrm{~B} \rightarrow \pi^{+} \pi^{-} \pi$ & $5.0 \times 10^{-5}$ & $<20$ \\
$\mathrm{~B}_{\mathrm{s}}^{0} \rightarrow \pi^{+} \mathrm{K}^{-}$ & $0.7 \times 10^{-5}$ & 600 \\
$\mathrm{~B}_{\mathrm{s}}^{0} \rightarrow \mathrm{K}^{+} \mathrm{K}^{-}$ & $1.5 \times 10^{-5}$ & 1300 \\
$\Lambda_{\mathrm{b}} \rightarrow \mathrm{p} \pi^{-}$ & $8.0 \times 10^{-5}$ & 600 \\
$\Lambda_{\mathrm{b}} \rightarrow \mathrm{pK}$ & $8.0 \times 10^{-5}$ & 1000 \\
$\mathrm{Comb}$. & - & 900 \\
\hline Total bkg & - & 9000 \\
\hline
\end{tabular}


the $>10^{7}$ rejection factor on the trigger Level 1 output.

The $\mathrm{B}_{\mathrm{d}}^{0} \rightarrow \mathrm{K}^{+} \pi^{-}$and $\pi^{+} \pi^{-}$branching ratios are recent, still imprecise first measurements from CLEO [9]. The other branching ratios have been inferred from the first two with simple SU(3) symmetry, except the $\Lambda_{\mathrm{b}}$ decays which have been set to their experimental upper limits [10].

The most abundant background is the $\mathrm{B}_{\mathrm{d}}^{0} \rightarrow \mathrm{K}^{+} \pi^{-}$decay, because of the absence of $\pi / \mathrm{K}$ separation, and because the mass resolution is higher than the separation between the peaks $\left(\sim 40 \mathrm{MeV} / \mathrm{c}^{2}\right)$ (see Fig. 2).

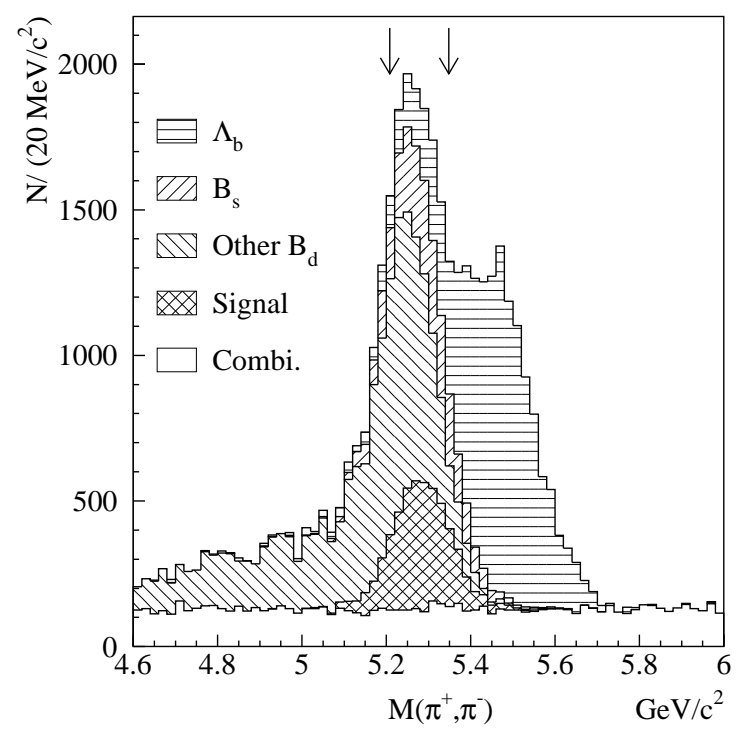

Figure 2. $\pi^{+} \pi^{-}$mass spectrum. The arrows indicate the signal window.

\subsection{Results}

The background dilution is $D_{b k g}=0.21$. Initial state tagging is done with the triggering muon, yielding a tagging dilution of $D_{t a g}=0.56$. The time-integrated method yields a dilution $D_{\text {int }}=0.63$. The statistical sensitivity to $\sin 2 \alpha$ is then, in the absence of penguin decays:

$\delta_{\text {stat }}(\sin 2 \alpha)=0.12$.

If the minimum pion $p_{\mathrm{T}}$ at trigger Level 2 cannot be lowered from 5.5 to $4 \mathrm{GeV} / \mathrm{c}$, the sensitivity degrades to 0.18 . Systematic uncertainties arising from asymmetry in the detection efficiency and from the lepton tagging are expected to be of order 0.01 , as in the $\mathrm{B}_{\mathrm{d}}^{0} \rightarrow \mathrm{J} / \psi \mathrm{K}_{\mathrm{S}}^{0}$ channel.

By year 2005, near-future B factories will have measured precisely the branching ratios of the two-body decay channels. A 5\% relative uncertainty on these branching ratios, will give a relative uncertainty on the asymmetry of less than $5 \%$. More problematic is the possibility that these backgrounds exhibit some CP asymmetry themselves (in fact the asymmetry in $\mathrm{B}_{\mathrm{d}}^{0} \rightarrow \mathrm{K}^{+} \pi^{-}$ could help constraint $\gamma[11])$. A more sophisticated fitting method (now under study) will have to be used, taking into account on a event by event basis the invariant mass and the lifetime. The $\Lambda_{\mathrm{b}}$ decays (which does not oscillate) and the $\mathrm{B}_{\mathrm{s}}^{0}$ decays (with rapid oscillation period $\sim 0.5 \mathrm{ps}$ ) will then be distinguished from $\mathrm{B}_{\mathrm{d}}^{0}$ decays (with slow oscillation period $\sim 14 \mathrm{ps}$ ). The $\mathrm{B}_{\mathrm{d}}^{0} \rightarrow \pi^{+} \pi^{-}$ decay and the $\mathrm{B}_{\mathrm{d}}^{0} \rightarrow \mathrm{K}^{+} \pi^{-}$decay will not be completely separated. ATLAS will measure a linear combination of the CP asymmetries in these decays, which will be combined with results from other experiments.

There is also a potential factor four increase of the statistics from the lowering of the minimum $p_{\mathrm{T}}$ of the pions from 4 to $1 \mathrm{GeV} / \mathrm{c}$ (now under study). This requires more sophisticated cuts to keep the combinatorial background and the trigger Level 2 rate low enough.

\section{4. $\delta \gamma:$ the $\mathrm{B}_{\mathrm{s}}^{\mathrm{o}} \rightarrow \mathrm{J} / \psi \phi$ channel}

If the decay $\mathrm{B}_{\mathrm{s}}^{0} \rightarrow \mathrm{J} / \psi \phi$ is a pure $\mathrm{CP}=+1$ amplitude, the asymmetry in the channel $\mathrm{B}_{\mathrm{s}}^{0} \rightarrow$ $\mathrm{J} / \psi \phi$ is:

$A(t)=\sin (2 \delta \gamma) \sin \left(x_{\mathrm{s}} t / \tau_{\mathrm{B}_{\mathrm{s}}^{0}}\right)$.

Dropping this assumption necessitates to introduce a new fit parameter, the ratio of the $\mathrm{CP}=-1$ and $\mathrm{CP}=+1$ amplitudes. Nevertheless, the sensitivity on the $\mathrm{CP}$ violating oscillation amplitude 
$A_{C P}$ is still a good measure of the sensitivity on $\delta \gamma . \delta \gamma$ is expected to be small $(\sim 0.01 \mathrm{rad})$ in the standard model. A high value would be a signature for new physics.

The measurement of this asymmetry is similar to the one for the $\mathrm{B}_{\mathrm{d}}^{0} \rightarrow \mathrm{J} / \psi \mathrm{K}_{\mathrm{S}}^{0}$ channel, except that the fast $\mathrm{B}_{\mathrm{s}}^{0}$ oscillations make it necessary to resolve them with a good proper-time resolution. With a resolution of $0.07 \mathrm{ps}$, the dilution of the asymmetry with a time-dependent fit is $D_{\text {fit }}=$ 0.47 , for $x_{\mathrm{s}}=20$ (the current standard model fit favoured value [6]).

The event selection is similar to the $\mathrm{B}_{\mathrm{d}}^{0} \rightarrow$ $\mathrm{J} / \psi \mathrm{K}_{\mathrm{S}}^{0}$ one. The absence of $\mathrm{K}$ identification is not a problem for the reconstruction of $\phi \rightarrow \mathrm{K}^{+} \mathrm{K}^{-}$. The yields are shown in Table 3 . All samples combined, the sensitivity to $A_{C P}$ is:

$\delta\left(A_{C P}\right)=0.027$.

The sensitivity varies from 0.025 to 0.045 if $x_{\mathrm{s}}$ varies in the currently allowed range 15 to 30 [6].

Table 3

Yields and sensitivity in the $\mathrm{J} / \psi \phi$ channel.

\begin{tabular}{ccc}
\hline Channel & $\mathrm{e}^{+} \mathrm{e}^{-}$ & $\mu^{+} \mu^{-}$ \\
\hline $\mathrm{N} \mathrm{B}_{\mathrm{s}}^{0} \rightarrow \mathrm{J} / \psi \phi$ & 3500 & 134000 \\
$\mathrm{~N}$ background & 200 & 3200 \\
\hline$\delta(A)$ lepton tag & 0.059 & 0.049 \\
$\delta(A)$ jet tag & 0.224 & 0.044 \\
\hline
\end{tabular}

\section{5. $\gamma$ : the $\mathbf{B}_{\mathrm{d}}^{0} \rightarrow \mathrm{D}_{(1)}^{0} \mathrm{~K}^{* 0}$ channel}

The set of the three decays $\mathrm{B}_{\mathrm{d}}^{0} \rightarrow \overline{\mathrm{D}}^{0} \mathrm{~K}^{* 0}$, $\mathrm{B}_{\mathrm{d}}^{0} \rightarrow \mathrm{D}^{0} \mathrm{~K}^{* 0}, \mathrm{~B}_{\mathrm{d}}^{0} \rightarrow \mathrm{D}_{1}^{0} \mathrm{~K}^{* 0}\left(\mathrm{D}_{1}^{0}\right.$ is the $\mathrm{D}^{0} \mathrm{CP}$ eigenstate, decaying to $\pi^{+} \pi^{-}$and $\mathrm{K}^{+} \mathrm{K}^{-}$) and their charge conjugate can be described by two triangles differing only by the side corresponding to the $\mathrm{CP}$ asymmetric decays $\mathrm{B}_{\mathrm{d}}^{0} \rightarrow \mathrm{D}_{1}^{0} \mathrm{~K}^{* 0}$ and $\overline{\mathrm{B}}_{\mathrm{d}}^{0} \rightarrow \mathrm{D}_{1}^{0} \overline{\mathrm{K}}^{* 0}$. The measurement of the six rates determined the angle $\gamma$. The current standard model best fit to the Unitarity Triangle yields $\gamma=(64 \pm 12)^{\circ}[6]$

No initial state tagging is necessary since the flavour of the $\mathrm{K}^{* 0}$ tags the flavour of the $\mathrm{B}_{\mathrm{d}}^{0}$. How- ever, ATLAS needs a high $p_{\mathrm{T}}$ muon for trigger Level 1. The decays $\mathrm{D}^{0} \rightarrow \mathrm{K}^{-} \pi^{+}, \overline{\mathrm{D}}^{0} \rightarrow \mathrm{K}^{+} \pi^{-}$, $\mathrm{D}^{0}$ or $\overline{\mathrm{D}}^{0} \rightarrow \mathrm{K}^{+} \mathrm{K}^{-}$or $\pi^{+} \pi^{-}$and $\mathrm{K}^{* 0} \rightarrow \mathrm{K}^{+} \pi^{-}$, $\overline{\mathrm{K}}^{* 0} \rightarrow \mathrm{K}^{-} \pi^{+}$must be separated without $\pi / \mathrm{K}$ separation. It is possible to veto all ambiguous events with an efficiency loss less than $50 \%$. It is also necessary to separate these events from the combinatorial background as only a yield of a few events a year is expected in some of these decays. Nevertheless, assuming a signal to background ratio of one (now under study), a sensitivity to $\gamma$ of $20^{\circ}$ in three years data-taking can be reached.

\section{Conclusion}

Improved analyses with full simulation of the final ATLAS detector show that ATLAS will have an excellent sensitivity on $\sin 2 \beta$ in the $\mathrm{J} / \psi \mathrm{K}_{\mathrm{S}}^{0}$ channel. The measurement of $\sin 2 \alpha$ in the $\mathrm{B}_{\mathrm{d}}^{0} \rightarrow$ $\pi^{+} \pi^{-}$channel will be difficult, but ATLAS will be able to contribute valuable information. Sensitivity to $\delta \gamma$ in the $\mathrm{B}_{\mathrm{s}}^{0} \rightarrow \mathrm{J} / \psi \phi$ channel is sufficient to allow a precise test of the standard model prediction. Measurement of $\gamma$ in the channels $\mathrm{B}_{\mathrm{d}}^{0} \rightarrow \mathrm{D}_{(1)}^{0} \mathrm{~K}^{* 0}$ may be feasible if rates are more favourable than current estimates.

\section{REFERENCES}

1. C. Caso et al.. (Particle Data Group), Eur. Phys. J. C3 (1998) 1.

2. P. Sherwood, these proceedings.

3. The ATLAS Collaboration, Technical Proposal, CERN/LHCC/94-43, 1994.

4. P. Eerola, Nucl. Instr. and Meth. A $\mathbf{3 8 4}$ (1996) 93.

5. G.F. Tartarelli, Nucl. Instr. and Meth. A 408 (1998) 110.

6. F. Parodi et al., hep-ph/9802289

7. K. Kelley, these proceedings.

8. R. Fleischer and T. Mannel, Phys. Lett. B397 (1997) 269.

9. R. Godang et al. (CLEO Collaboration), Phys. Rev. Lett. 80 (1998) 3456.

10. D. Buskulic et al. (ALEPH Collaboration), Phys. Lett. B384 (1996) 471.

11. R. Fleischer, CERN-TH/98-60, to appear in Eur. Phys. J. C. 